\title{
Self-consistent field theory for lipid-based liquid crystals: Hydrogen bonding effect
}

\author{
Won Bo Lee, ${ }^{1}$ Raffaele Mezzenga, ${ }^{2,3, a), b)}$ and Glenn H. Fredrickson ${ }^{4, a), c)}$ \\ ${ }^{1}$ Department of Chemical Engineering, University of California, Santa Barbara, California 93106, USA \\ ${ }^{2}$ Department of Physics, University of Fribourg, Perolles, Fribourg CH-1700, Switzerland \\ ${ }^{3}$ Nestlé Research Center, Vers-Chez-Les-Blanc, 1000 Lausanne 26, Switzerland \\ ${ }^{4}$ Department of Chemical Engineering and Materials and Materials Research Laboratory, \\ University of California, Santa Barbara, California 93106, USA
}

\begin{abstract}
A model to describe the self-assembly properties of aqueous blends of nonionic lipids is developed in the framework of self-consistent field theory (SCFT). Thermally reversible hydrogen bonding between lipid heads and water turns out to be a key factor in describing the lyotropic and thermotropic phase behavior of such systems. Our model includes reversible hydrogen bonding imposed in the context of the grand canonical ensemble and exact conditions of binding equilibrium. The lipid molecules are modeled as a rigid head and a flexible Gaussian tail, and the water molecules are treated explicitly. Here, we focus on systems where the lipid molecule has a relatively small hydrophilic head compared to the hydrophobic tail, such as monoolein in water. Experimentally, this system has both normal phase sequences (inverted hexagonal to inverted double gyroid cubic phase) and reverse phase sequences (lamellar to inverted double gyroid cubic phase) as the water volume fraction increases. From SCFT simulations of the model, two phase diagrams corresponding to temperature independent or dependent interaction parameters $\chi$ are constructed, which qualitatively capture the phase behavior of the monoolein-water mixture. The lattice parameters of the simulated mesophases are compared with the experimental values and are found to be in semiquantitative agreement. The role of various structural and solution parameters on the phase diagrams is also discussed.
\end{abstract}

\section{INTRODUCTION}

The mesophases found in lipid-water mixtures have received attention in a number of areas such as cosmetic, food, and pharmaceutical formulations for the delivery of flavors, drugs, and aromas. ${ }^{1-4}$ In order to understand the various structures and the phase behavior of the lipid-water mixtures, a series of experiments has been performed in the past that explore both thermal and concentration effects. Of particular interest is the placement and stability of cubic bicontinuous phases, as these provide continuous pathways for transport of flavors and fragrances in both hydrophobic and hydrophilic domains. Such phases have been observed and studied via small angle $\mathrm{x}$-ray scattering and transmission electron microscopy. ${ }^{5,6}$ One system that has been particularly well studied is the mixture of monoolein with water. As shown in Fig. 1(a), monoolein has a monounsaturated lipid tail and a head group that can hydrogen bond with up to ten water molecules. The monoolein-water system has been shown to exhibit two different (triply periodic) bicontinuous cubic phases: ${ }^{7,8}$ The "double gyroid" cubic phase $(I a \overline{3} d$ space group) and the "double diamond" cubic phase $(P n \overline{3} m$ space group). Both of these structures are so-called "inverted"

\footnotetext{
a) Authors to whom correspondence should be addressed.

b) Electronic mail: raffaele.mezzenga@unifr.ch.

${ }^{c)}$ Electronic mail: ghf@mrl.ucsb.edu.
}

phases in the sense that water fills the percolating network of cylindrical struts, while the oily tails fill the connected space outside the struts. The first phase diagram of the monooleinwater system was produced by Hyde et al. ${ }^{9}$ and a more complete phase diagram was constructed by Caffrey and co-workers. ${ }^{10,11}$ Several other closely related nonionic lipid systems, such as monovaccenin and monononadecenoin, have also been studied. ${ }^{12,13}$

In spite of these experimental studies, there is limited understanding of how molecular-level factors in the monoolein class of lipid-water mixtures influence mesoscale self-assembly and phase behavior. An outstanding puzzle is that these systems have both a normal phase sequence from the inverted hexagonal phase $\left(H_{\mathrm{II}}\right)$ to the inverted gyroid phase $\left(I a \overline{3} d_{\mathrm{II}}\right)$ and a reverse phase sequence from the lamellar phase $\left(L_{\alpha}\right)$ to the gyroid phase $\left(I a \overline{3}_{\text {II }}\right)$ as the water volume fraction is increased. Prior to our work, there has been no molecular-based theoretical model that even qualitatively describes both of these lyotropic sequences while simultaneously capturing the thermotropic phase behavior of the monoolein class of water-lipid mixtures. Evidently, there are a number of physical ingredients that should be considered in building such a model. These include hydrogen bonding between the lipid head and surrounding water, the hydrophobic effect, the length of the hydrocarbon tail, the dependence of the flexibility of the tail on the degree of unsaturation, and 


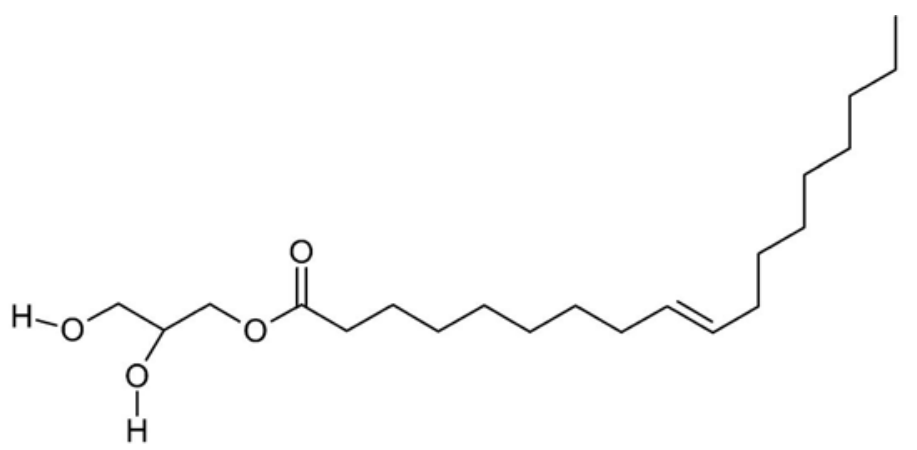

(b)
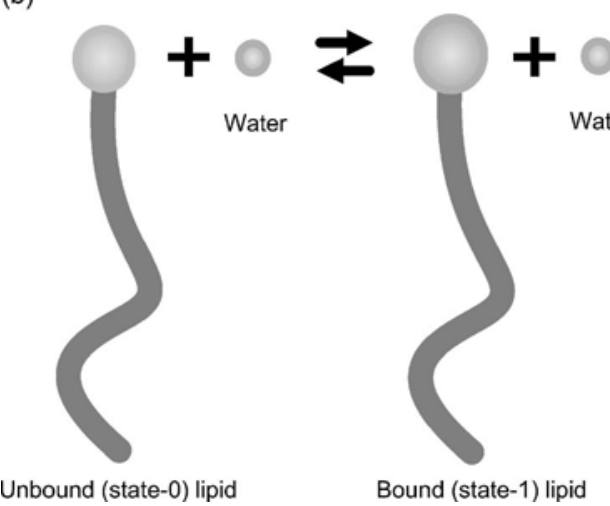

Bound (state-1) lipid
FIG. 1. (a) Chemical structure of a monoolein molecule. (b) Schematic representation of our $(k+1)$-state lipid hydration model. Upon binding with water the lipid head group grows in volume. among others. In the present paper, we show that a successful model can be constructed by focusing on the hydrogen bonding effect with concomitant changes in head group volume and interactions with the surrounding environment.

Previous theoretical work in the field provides some insights on how to proceed. A simple and useful tool for understanding membrane curvature and the stability of various lipid and surfactant phases in solution is based on the concept of a critical packing parameter $(\mathrm{CCP}),{ }^{14}$ which is defined as the ratio of the volume of a lipid tail to the product of the cross-sectional area of a lipid head and the length of a lipid molecule. The CCP approach is widely used to explain the normal phase sequences observed in a variety of surfactant water systems. However, it does not provide any explanation for a reverse phase sequence such as the $L_{\alpha} \rightarrow I a \overline{3} d_{\mathrm{II}}$ transition observed in the monoolein-water system.

An elasticity model initiated by Canham, ${ }^{15}$ and Helfrich $^{16}$ is another type of phenomenological model that describes the membranes formed by lipid bilayers as thin elastic sheets characterized by material parameters such as spontaneous curvature, bending rigidity, and saddle splay modulus. In this curvature model, all molecular details are subsumed in the phenomenological material parameters. While the model does produce a rich set of morphologies including lamellar and various cubic phases, ${ }^{17}$ it is based on a weak curvature expansion and is of limited utility for typical lyotropic mesophases where the membrane curvature is large. Moreover, the model does not provide any insights into the relationship between the membrane parameters and the molecular details of a lipid-water system.

At the other extreme, fully atomistic simulations have been attempted for describing membrane and mesophase structures. ${ }^{18,19}$ This class of models preserves the full chemi- cal details of the lipid molecules and includes all types of interactions such as intramolecular (stretching, bending, and torsional), van der Waals, and electrostatic. The potential parameters are evaluated by $a b$ initio calculations or from experimental data. Wilson and Pohorille ${ }^{18}$ studied a lipid-water bilayer using molecular dynamics with potential parameters evaluated via $a b$ initio simulations and obtained bilayer properties, which agree with experimentally measured values. Recently, there was an initial promising attempt at stabilizing the bicontinuous double diamond cubic $(P n \overline{3} m)$ phase for the monoolein-water system. ${ }^{19}$ However, the stateof-the-art molecular simulations are restricted to very small computational cells and of the order of $10 \mathrm{~ns}$ of simulated trajectories. These restrictions effectively limit atomistic simulation studies to relaxation of seeded initial configurations $^{20,21}$ since the time scales of self-assembly in lipid-water mixtures are many orders of magnitude larger than the 10 ns simulation window.

Self-consistent field theory (SCFT) is a coarse-grained field-theoretic model solved within the mean field approximation. SCFT provides a dramatic computational advantage compared to fully atomistic approaches, ${ }^{22,23}$ but still captures the gross architectural details of molecules and their interactions. It is a powerful and flexible method that has been successfully applied to study the phase behavior of a wide variety of inhomogeneous polymer systems such as melts, blends, and solutions. ${ }^{23-27} \mathrm{Li}$ and Schick $^{28}$ have shown that SCFT models of lipids with flexible Gaussian tails and rigid polar heads can reproduce normal phase sequences (including cubic phases) and their dependence on the relative volumes of head and tail for a neutral lipid. They also incorporated a hydration effect caused by electrostatic interaction between the charge of lipid head and the dipole of water to 
treat charged lipid systems. The use of flexible Gaussian chain model for a short lipid tail provides computational advantage while it approximates the tail entropy. However, it still provides reasonable approximation for the lipid tail in the liquid crystalline state, ${ }^{29}$ although it does not correctly account for the tail entropy in the solid crystalline state. A more sophisticated SCFT model proposed by Müller and Schick uses the rotational isomeric state model to represent lipid tail conformations. ${ }^{30}$ However, these SCFT studies, which did not allow for hydrogen bond formation, did not reproduce the reverse lyotropic phase sequences that dominate the monoolein-water phase diagram.

In a recent letter, we reported on a field-theoretic model whose mean field (SCFT) solution captures the salient features of the monoolein-water phase diagram. ${ }^{31}$ Here, we expand on the details of the model and the computational methods employed and provide a broader set of simulation results. We also compare our theoretical predictions for lattice constants of the mesophases with the literature values obtained from small angle x-ray scattering experiments.

\section{MODEL}

In the present paper, we introduce a simple field theory model that we believe captures the essential thermotropic and lyotropic physics of lipid-water mixtures in the monoolein class. As shown in Fig. 1(a), monoolein has a monounsaturated lipid tail and a head group that can hydrogen bond with up to ten water molecules. In the coarse-grained description depicted in Fig. 1(b), we simplify the molecule to a flexible Gaussian chain tail consisting of $N$ segments, each of volume $v_{t}$, and a rigid head that can exist in one of $k+1$ states: State "0," which has no bound water and a small head volume $v_{h 0}$, and state " $j "(j=1, \ldots, k)$, which has $j$ bound waters and a larger head volume $v_{h j}$. The thermally reversible binding of water to the head is one key component of the model, which also produces changes in the head-water and the head-tail interactions, as will be described below. The experimental phase diagrams of monoolein ${ }^{9,11}$ and monolinolein $^{32}$ systems show a phase transition from fluid isotropic to lamellar phases $\left(F I \rightarrow L_{\alpha}\right)$ at low temperature $\left(\sim 40^{\circ} \mathrm{C}\right)$ and low water concentration $[\sim 6 \%(w / w)]$. Therefore, the head-tail interaction in the pure (unbound) lipids is not strong enough to produce an ordered phase such as $L_{\alpha}$ phase. As the water concentration is increased at low temperature, we expect that these systems bind all the available water and are composed of a mixture of unbound and bound lipid molecules. Since, experimentally, the $L_{\alpha}$ phase appears before all the lipid heads are saturated with water and a significant amount of free water is present, we conclude that lipid heads with bound water must interact less favorably with tail segments than unbound heads. In the context of our model, we thus build in interactions such that the larger state $j>0$ hydrated head should interact less favorably with free (unbound) water and lipid tails than a head in the dry state 0 .

Free water in the model is treated as a space-filling pointlike species. Local incompressibility of the solution is imposed, so that the volume-weighted sum of all species densities is constrained to a constant value throughout the system. The total system volume is denoted by $V$. The nonbonded interactions among the various species are described by Flory-Huggins $\chi$ parameters. The energy change (in units of the thermal energy $k_{B} T$ ) associated with incremental hydrogen bonding of the head group (from state $j-1$ to state $j$ ) is denoted by $F_{b}$. Subscripts $h j, w$, and $t$ will be used throughout to denote, respectively, lipid heads in hydration state $j$, free water, and lipid tails, respectively.

In the continuous Gaussian chain model, conformations of noninteracting lipids have a probability proportional to a Gaussian statistical weight $e^{-\beta U_{0}}$, where $\beta U_{0}$ is the harmonic stretching energy of lipids,

$$
\beta U_{0}=\frac{1}{4 R_{g}^{2}} \sum_{j=0}^{k} \sum_{i=1}^{n_{j}} \int_{0}^{1} d s\left|\frac{d \mathbf{r}_{j, i}(s)}{d s}\right|^{2}
$$

In this expression, $k$ represents the number of bound lipid states and $n_{j}$ is the number of state $j$ lipid molecules in the system. $R_{g}=b \sqrt{N / 6}$ is the unperturbed radius of gyration of a lipid tail ( $b$ is the tail statistical segment length) and $\mathbf{r}_{j, i}(s)$ is a continuous space curve describing the shape of the $i^{\text {th }}$ lipid in state $j$. Nonbonded interactions are included by analogy with the Flory-Huggins lattice theory through appropriate $\chi$ parameters,

$$
\beta U_{1}=\rho_{0} \int d \mathbf{r} \sum_{l<m} \chi_{l, m} \hat{\rho}_{l} \hat{\rho}_{m}
$$

where $\rho_{0}$ is a reference constant segment density, which we fix equal to the pure state density of water, $1 / v_{w}$. The quantities $\hat{\rho}_{l}$ are microscopic reduced densities (volume fractions) of species $l=(h j, t, w)$ defined by

$$
\begin{aligned}
& \hat{\rho}_{h j}=\sum_{i=1}^{n_{j}} v_{h j} \delta\left(\mathbf{r}-\mathbf{r}_{j, i}(1)\right), \\
& \hat{\rho}_{t}=v_{t} N \sum_{j=0}^{k} \sum_{i=1}^{n_{j}} \int_{0}^{1} d s \delta\left(\mathbf{r}-\mathbf{r}_{j, i}(s)\right), \\
& \hat{\rho}_{w}=v_{w} \sum_{i=1}^{n_{w}} \delta\left(\mathbf{r}-\mathbf{r}_{w}\right) .
\end{aligned}
$$

The objects $\hat{\rho}_{h j}, \hat{\rho}_{t}$, and $\hat{\rho}_{w}$ are the microscopic volume fractions contributed by head groups in hydration state $j$, lipid tails whose heads are in any hydration state, and free (unbound) water, respectively. The total head group microscopic volume fraction (irrespective of hydration state) is $\hat{\rho}_{h}$ $=\sum_{j=0}^{k} \hat{\rho}_{h j}$.

Based on earlier studies of supramolecular polymer assembly, ${ }^{33,34}$ we have found that field theory models with reversible binding (or other chemical reaction equilibria) are most conveniently formulated in the grand canonical ensemble. The relevant grand canonical partition function for the lipid-water model just described is given by 


$$
\begin{aligned}
\Xi= & \sum_{n_{0}=0}^{\infty} \cdots \sum_{n_{k}=0}^{\infty} \sum_{n_{w}=0}^{\infty} \frac{\left(z_{w}\right)^{n_{w}}}{n_{w} !} \prod_{j=0}^{k} \frac{1}{n_{j} !}\left[\frac{k ! z_{j}}{(k-j) ! j !}\right]^{n_{j}} \\
& \times \prod_{j=0}^{k} \prod_{i=1}^{n_{j}} \int \mathcal{D} \mathbf{r}_{j, i} \prod_{l=1}^{n_{w}} \int d \mathbf{r}_{l} e^{-\beta U_{0}-\beta U_{1}} \\
& \times \delta\left[\hat{\rho}_{h}+\hat{\rho}_{t}+\hat{\rho}_{w}-1\right]
\end{aligned}
$$

where $\int \mathcal{D} \mathbf{r}_{j, i}$ denotes a path integral over the tail conformations of the $i^{\text {th }}$ lipid that is in hydration state $j$ and $\int d \mathbf{r}_{l}$ denotes a volume integral over the position of the $l^{\text {th }}$ water molecule. The object $\delta\left[\hat{\rho}_{h}+\hat{\rho}_{t}+\hat{\rho}_{w}-1\right]$ is a Dirac delta functional that imposes a local incompressibility constraint on the fluid, namely, that the sum of all microscopic segment volume fractions must equal to unity at all positions within the volume $V$. The free water activity is denoted of $z_{w}$, while $z_{j}$ is the activity of lipids that are in hydration state $j$. For the free water species, a factor of $1 / n_{w}$ ! accounts for the indistinguishability of the $n_{w}$ molecules, while a factor of

$$
\frac{1}{n_{j} !}\left[\frac{k !}{(k-j) ! j !}\right]^{n_{j}}
$$

simultaneously accounts for the indistinguishability of the lipids in hydration state $j$ and for the number of ways of binding $j$ water molecules at a total of $k$ identical head sites on each lipid.

Obviously, this "particle-based" partition function cannot be solved analytically. However, the partition function can be transformed into the form of a statistical field theory by means of some well known steps. Specifically, we invoke the identity

$$
\begin{aligned}
1 & =\int \mathcal{D} \phi \delta[\phi-\hat{\rho}] \\
& =\int \mathcal{D} \phi \int \mathcal{D} w \exp \left[\int \operatorname{driw}(\mathbf{r})(\phi(\mathbf{r})-\hat{\rho}(\mathbf{r}))\right],
\end{aligned}
$$

where $\int \mathcal{D} \phi$ and $\int \mathcal{D} w$ denote functional integrals over a volume fraction field $\phi(\mathbf{r})$ and an auxiliary potential field $w(\mathbf{r})$, respectively. The incompressibility constraint in Eq. (4) can be similarly expressed as

$$
\begin{aligned}
\delta\left[\hat{\rho}_{h}\right. & \left.+\hat{\rho}_{t}+\hat{\rho}_{w}-1\right] \\
& =\int \mathcal{D} p \exp \left[\int \operatorname{drip}\left(\hat{\rho}_{h}+\hat{\rho}_{t}+\hat{\rho}_{w}-1\right)\right],
\end{aligned}
$$

where $p(\mathbf{r})$ is a fluctuating pressure field. By inserting the above expressions into Eq. (4) and rescaling fields according to $W_{j}=i N w_{j}$ and $P=i N p(i=\sqrt{-1})$, the grand canonical partition function can be written in the following field-theoretic form: $:^{23,24}$

$$
\Xi \sim \int \mathcal{D} \mathbf{W} \int \mathcal{D} \phi \int \mathcal{D} P e^{-\beta H_{G}[\mathbf{W}, \boldsymbol{\phi}, P]},
$$

where $\boldsymbol{\phi}$ and $\mathbf{W}$ are $(k+3)$-component vector fields representing the volume fractions and auxiliary potentials of the $h j, w$, and $t$ species, respectively. The "effective Hamiltonian" appearing in the above field theory can be expressed as

$$
\begin{aligned}
h_{G} \equiv \frac{\beta N H_{G}}{\rho_{0} V}= & -z_{w} Q_{w}-\sum_{j=0}^{k} g_{j, k} z_{h j} Q_{h j} \\
& +\frac{1}{V} \int d \mathbf{r}\left\{\sum _ { j = 0 } ^ { k } \left(\chi_{h j, t} N \phi_{h j} \phi_{t}\right.\right. \\
& \left.+\chi_{h j, w} N \phi_{h j} \phi_{w}\right)+\chi_{t, w} N \phi_{t} \phi_{w} \\
& -\left(\sum_{j=0}^{k} W_{h j} \phi_{h j}+W_{t} \phi_{t}+W_{w} \phi_{w}\right) \\
& \left.+P\left(\sum_{j=0}^{k} \phi_{h j}+\phi_{t}+\phi_{w}-1\right)\right\},
\end{aligned}
$$

where $g_{j, k} \equiv k ! /[(k-j) ! j !]$ is the binding site degeneracy factor noted above. The objects $Q_{w}$ and $Q_{h j}$ are the partition functions for a single water molecule and a single state $j$ lipid experiencing potential fields, which are linear combinations of $\mathbf{W}$ and $P$. Explicit expressions for these objects, which are evidently functionals of $\mathbf{W}$ and $P$, will be given below.

A few comments are in order with regard to the form of Eq. (8). First, all activities have been expressed in dimensionless form by normalizing them by a reference density $\rho_{0} / N=1 /\left(v_{w} N\right)$. Furthermore, due to the incompressibility constraint, one of the species' activities can be freely specified. We have therefore arbitrarily set $z_{w}=1$. The equilibrium constraints associated with the water binding reactions have also not yet been imposed. The conditions of reaction equilibria for our $(k+1)$-state model of hydrogen bonding corresponds to the following $k$ constraints on the activities: ${ }^{33,34}$

$$
\frac{z_{h j+1}}{z_{w} z_{h j}}=\exp \left(-F_{b}\right) / N, \quad j=0,1, \ldots, k-1 .
$$

Even after transformation to a field-theoretic form, the partition function of Eq. (7) still cannot be solved analytically in closed form, so numerical methods and/or analytical approximations are required. In this paper, we report only on mean-field, or SCFT, solutions of the field theory. The meanfield equations result from demanding that $h_{G}$ be stationary with respect to variations of the $2 k+7$ field components $\left(\delta h_{G} / \delta \mathbf{W}=\delta h_{G} / \delta \phi=\delta h_{G} / \delta P=0\right)$,

$$
\begin{aligned}
& \phi_{h j}=-g_{j, k} z_{h j} \frac{\delta Q_{h j}}{\delta W_{h j}}, \\
& \phi_{t}=-\sum_{j=0}^{k} g_{j, k} z_{h j} \frac{\delta Q_{h j}}{\delta W_{t}}, \\
& \phi_{w}=-\frac{\delta Q_{w}}{\delta W_{w}}, \\
& 0=\sum_{j=0}^{k} \phi_{h j}+\phi_{t}+\phi_{w}-1, \\
& W_{h j}=\chi_{h j, t} N \phi_{t}+\chi_{h j, w} N \phi_{w}+P,
\end{aligned}
$$




$$
\begin{aligned}
& W_{t}=\sum_{j=0}^{k} \chi_{h j, t} N \phi_{h j}+\chi_{t, w} N \phi_{w}+P, \\
& W_{w}=\sum_{j=0}^{k} \chi_{h j, w} N \phi_{h j}+\chi_{t, w} N \phi_{t}+P .
\end{aligned}
$$

The functional derivatives in the first three equations can be made explicit through the formulas ${ }^{23,25}$

$$
\begin{aligned}
& \phi_{h j}(\mathbf{r})=g_{j, k} z_{h j} \frac{v_{h j}}{N v_{w}} q_{h j}(\mathbf{r}, 0) q_{t}(\mathbf{r}, 1), \\
& \phi_{t}(\mathbf{r})=\frac{v_{t}}{v_{w}} \int_{0}^{1} d s \sum_{j=0}^{k} g_{j, k} z_{h j} q_{h j}(\mathbf{r}, s) q_{t}(\mathbf{r}, 1-s),
\end{aligned}
$$

and

$$
\phi_{w}(\mathbf{r})=(1 / N) e^{-W_{w}(\mathbf{r}) / N}
$$

The objects $q_{h j}(\mathbf{r}, s)$ and $q_{t}(\mathbf{r}, s)$ denote forward (starting from the head) and backward (starting from the free tail end) propagators for the conformations of a lipid in state $j$. The forward propagator $q_{h j}$ satisfies the diffusion equation

$$
\frac{\partial q_{h j}(\mathbf{r}, s)}{\partial s}=R_{g}^{2} \nabla^{2} q_{h j}(\mathbf{r}, s)-\frac{v_{t}}{v_{w}} W_{t}(\mathbf{r}) q_{h j}(\mathbf{r}, s)
$$

subject to the initial condition $q_{h j}(\mathbf{r}, 0)=e^{-v_{h j} / N v_{w} W_{h j}(\mathbf{r})}$. The reverse propagator $q_{t}$ (from the end of the lipid tail) satisfies the same diffusion equation, but with initial condition $q_{t}(\mathbf{r}, 0)=1$. Having the propagators introduced, the singlemolecule partition functions can be straightforwardly expressed as

$$
\begin{aligned}
Q_{h j} & =\frac{1}{V} \int d \mathbf{r} e^{-v_{h j} / N v_{w} W_{h j}(\mathbf{r})} q_{t}(\mathbf{r}, 1), \\
Q_{w} & =\frac{1}{V} \int d \mathbf{r} e^{-W_{w}(\mathbf{r}) / N} .
\end{aligned}
$$

The total water volume fraction $\phi_{w, \text { tot }}$ is defined as the sum of the volume fractions of free and bound water molecules and is calculated by the following formula:

$$
\phi_{w, \text { tot }}=\phi_{w}+\sum_{j=1}^{k} \frac{j v_{w}}{v_{h j}} \phi_{h j} .
$$

The above SCFT equations, [Eqs. (10)-(16)] represent a formidable set of nonlocal and nonlinear field equations. Nonetheless, powerful techniques have been devised to solve such equations in both a unit cell and large cell context. ${ }^{23,24}$ In the present work, initial field configurations are seeded in order to produce a unit cell of a desired mesophase, and the diffusion equations [Eq. (20)] (corresponding to $j$ $=0,1, \ldots, k$ and the additional equation for $q_{t}$ ) are solved pseudospectrally with an operator splitting scheme. ${ }^{35,36}$ From the calculated propagators, the volume fraction fields and single-molecule partition functions are evaluated using appropriate quadrature routines. The $\mathbf{W}$ and $P$ fields are subsequently relaxed using various convergence schemes ${ }^{23,37,38}$ in order to satisfy the remaining SCFT equations, [Eqs.
(13)-(16)]. Simultaneously, $h_{G}$ is minimized with respect to the size and the shape of the unit cell by a variable cell shape method $^{23,39}$ to eliminate any residual stress in the computational cell. The implementation of the variable cell shape method to the present model is outlined in the Appendix. Phase boundaries are determined by comparing the SCFT free energy densities, $h_{G}$, among phases at the same value of the activity of a 0 -state lipid, $z_{h 0}$.

For the general $(k+1)$-state model, the SCFT equations constitute $2 k+7$ equations for $2 k+7$ coupled fields. In the remainder of this paper, we consider only the simplest nontrivial two-state model corresponding to the particular choice of $k=1$. This choice retains what we believe to be the essential physics at play in the monoolein phase diagram, yet still leaves a formidable set of nine SCFT equations.

\section{RESULTS AND DISCUSSION}

\section{A. Mean-field phase diagrams}

Figure 2(a) shows the mean-field phase diagram of the two-state model with $v_{h 0} / v_{w}=1.8, v_{t} / v_{w}=1.0, N=5$, and the fixed $\chi$ parameter values indicated in the caption. The ordinate of Fig. 2(a), $-1 / F_{b}$, can be interpreted as a dimensionless temperature (assuming that hydrogen bonding is purely enthalpic), while the abscissa $\phi_{w, \text { tot }}$ is the total volume fraction of water (bound and free). (More accurately, the hydrogen bond interaction, $-F_{b}$, has an entropic contribution due to the directionality of the bond, ${ }^{40}$ which reduces the effective bonding strength.) The figure can thus be viewed as a first theoretical approximation to an experimental temperature versus water composition phase diagram.

A more realistic phase diagram can be constructed by taking into account the expected temperature dependence of the Flory $\chi$ parameters. In Fig. 2(b), we have generated a second mean-field phase diagram, in which we have assumed a conventional temperature dependence of the tail-water and hydrogen bond interactions, $\chi_{t, w} N=A+B / T,-F_{b}=C / T$, and fixed the ratio of bonding to interaction energy $C / B=0.5$. $A$ is determined by the choice of a reference set of interaction and bonding parameters specified in the figure caption, and the other $\chi_{i, j}$ are determined away from the reference point by maintaining constant values of the ratio $\chi_{i, j} / \chi_{t, w}$. To capture the gross topology of the monoolein-water phase diagram, it is important to choose the reference point $\chi$ parameters such that they have a large unfavorable "hydrophobic" interaction between tail and solvent $\chi_{t, w}$ and a head-solvent interaction that is initially small and becomes less favorable upon binding with water, i.e., $\chi_{h 0, w}<\chi_{h 1, w} \ll \chi_{t, w}$.

The SCFT phase diagrams [Figs. 2(a) and 2(b)] reproduce important qualitative features seen in the experimental diagram. ${ }^{11}$ For instance, they provide for a $L_{\alpha} \rightarrow I a \overline{3} d_{\mathrm{II}}$ $\rightarrow H_{\text {II }}$ phase sequence upon increasing temperature and a $F I \rightarrow L_{\alpha} \rightarrow I a \overline{3} d_{\text {II }}$ phase sequence upon increasing water volume fraction. The first sequence can be understood by referring to Figs. 3(a) and 4(a), which show how the composition of the fluid mixture changes along thermotropic trajectories in Figs. 2(a) and 2(b) at $\phi_{w, \text { tot }}=0.150$ and $\phi_{w, \text { tot }}=0.175$, respectively. As the temperature increases, the volume fraction of lipids with bound water decreases, and the effective size 


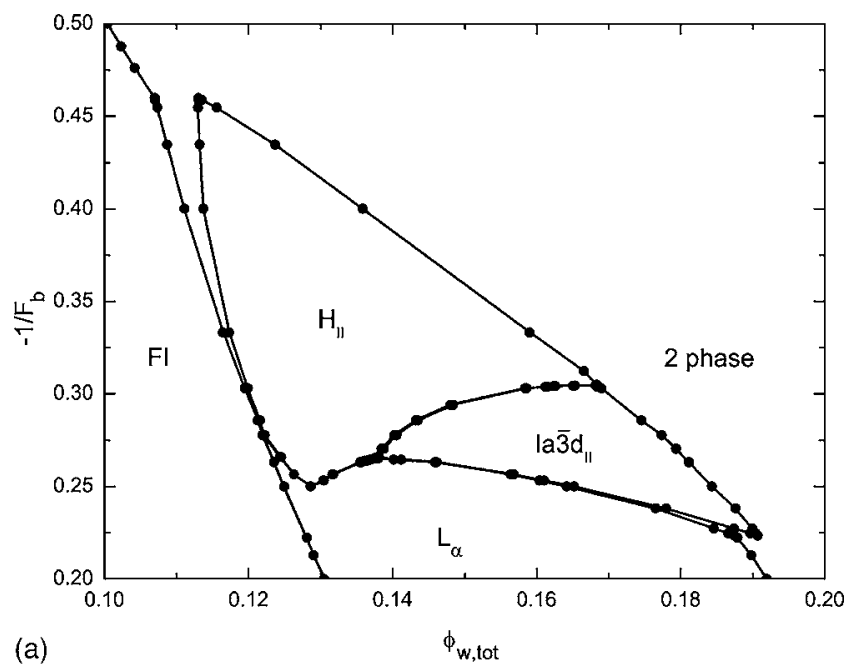

(a)

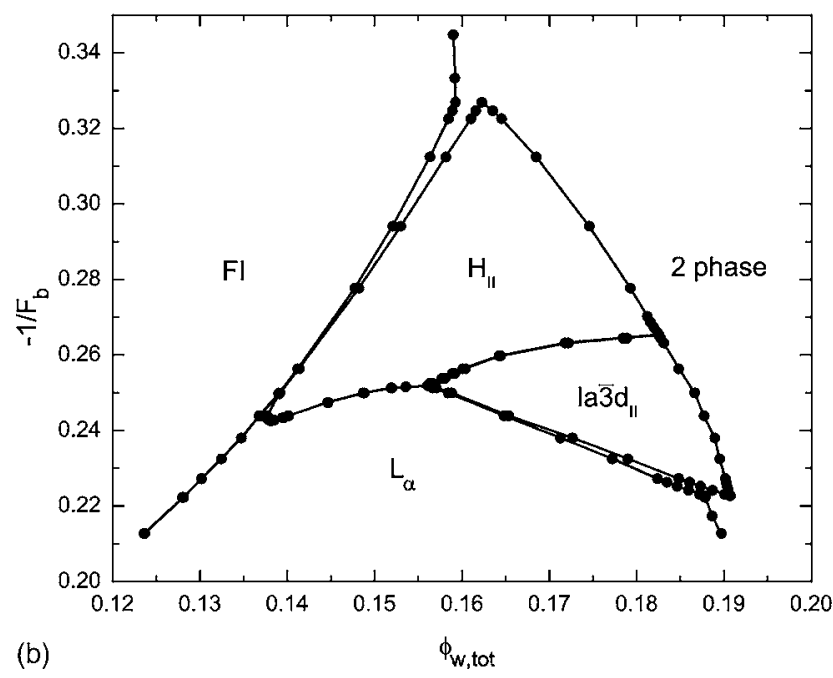

FIG. 2. (a) Mean-field phase diagram for the two-state model with $v_{h 0} / v_{w}$ $=1.8, v_{t} / v_{w}=1.0, N=5, \chi_{h 0, t}=1.0, \chi_{h 1, t}=1.45, \chi_{h 0, w}=0.1, \chi_{h 1, w}=0.5$, and $\chi_{t, w}=4.2$. The interaction parameters $\chi_{i, j}$ are independent of temperature. The labeled phases are FI (lipid-rich "fluid isotropic" homogenous phase), $H_{\mathrm{II}}$ (inverted hexagonal phase), $L_{\alpha}$ (lamellar phase), Ia $\overline{3} d_{\mathrm{II}}$ (inverted gyroid cubic phase), and two phase (region of coexistence with nearly pure water) (b) Mean-field phase diagram for the two-state model with $v_{h 0} / v_{w}=1.8$, $v_{t} / v_{w}=1.0, N=5$, and $C / B=0.5$, which allows the interaction parameters $\chi_{i, j}$ to change according to temperature. The reference point is $-F_{b}=4.5, \chi_{h 0,}$ $=1.0, \chi_{h 1, t}=1.45, \chi_{h 0, w}=0.1, \chi_{h 1, w}=0.5$, and $\chi_{t, w}=4.2$. The ratio $\chi_{i, j} / \chi_{t, w}$ is maintained constant over all values of $-F_{b}$.

of the lipid heads also decreases. A smaller lipid head stabilizes mesophases that curve toward water, e.g., the $H_{\text {II }}$ phase, in order to maximize the conformational entropy of the lipid tails. The second sequence shows the "reverse" lyotropic behavior present in the monoolein/water system. The volume fractions of the various species at a fixed value of $-F_{b}$ are shown in Figs. 3(b) [corresponding to the phase diagram in Fig. 2(a)] and 4(a) [corresponding to the phase diagram in Fig. 2(b)]. In particular, the $L_{\alpha} \rightarrow I a \overline{3} d_{\text {II }}$ phase transition occurs with increasing water volume fraction. As shown in Figs. 3(b) and 4(b), the $L_{\alpha}$ phase is stable when the lipids with bound water are saturated and the free water fraction is low, which is also consistent with the experimental phase diagrams. ${ }^{11,32}$ However, as more free water is present, the strong hydrophobic interaction between lipid tails and free
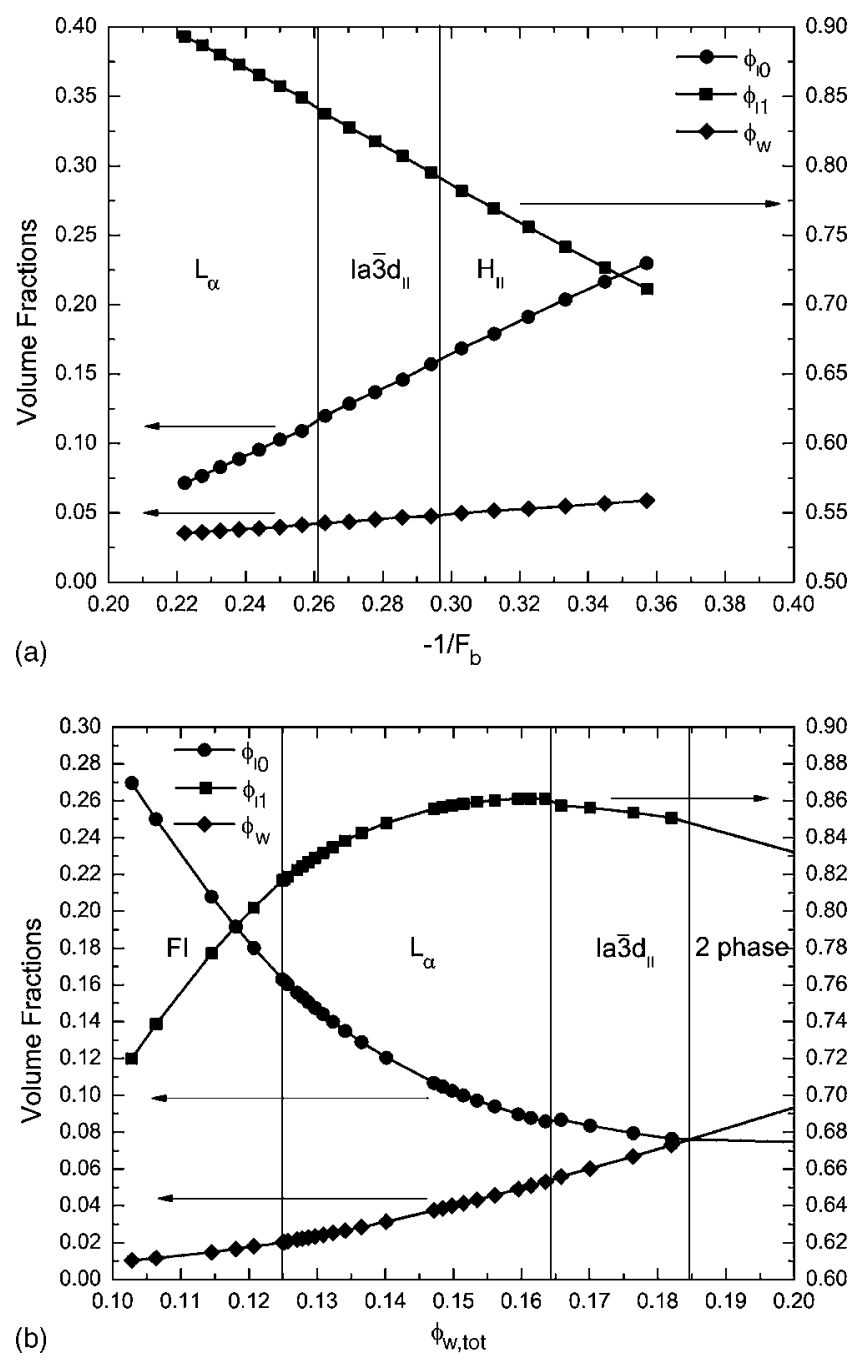

FIG. 3. (a) Volume fractions of free water $\left(\phi_{w}\right)$, unbound lipids $\left(\phi_{l 0}\right)$, and bound lipids $\left(\phi_{l 1}\right)$ vs $-1 / F_{b}$ at the fixed total water content of $\phi_{w \text {,tot }}$ $=0.150 \pm 0.0003$ and using the model parameters of Fig. 2(a). (b) Volume fractions vs $\phi_{w, \text { tot }}$ at fixed $-F_{b}=4.0$ and using the model parameters of Fig. 2(a).

water is more pronounced and drives a morphological transition to $I a \overline{3} d_{\mathrm{II}}$, wherein the interfacial area between hydrophilic and hydrophobic segments is reduced. The transition is further assisted by an increase in tail entropy. ${ }^{41}$

For the parameters chosen, the stability region of the $H_{\mathrm{II}}$ phase in Fig. 2(a), is exaggerated relative to the experimental phase diagram. However, as shown in Fig. 2(b), varying the $\chi$ parameters inversely with temperature rather than keeping them as fixed parameters, reduces the size of the $H_{\mathrm{II}}$ region. This procedure also transforms the negative slope of the phase boundary between $F I$ and $H_{\mathrm{II}}$ in Fig. 2(a) to a positive slope in Fig. 2(b), which is more consistent with the experimental phase diagram. ${ }^{11}$ The change in slope occurs in Fig. 2(b) because the incompatibility increases as temperature decreases, hence, stabilizing structured mesophases at lower temperature.

We note that the inverted double diamond cubic phase $\left(\operatorname{Pn} \overline{3} m_{\mathrm{II}}\right)$ is always metastable in the two phase diagrams for the parameters that we employed, although it most closely competes with the other mesophases just to the right (in wa- 

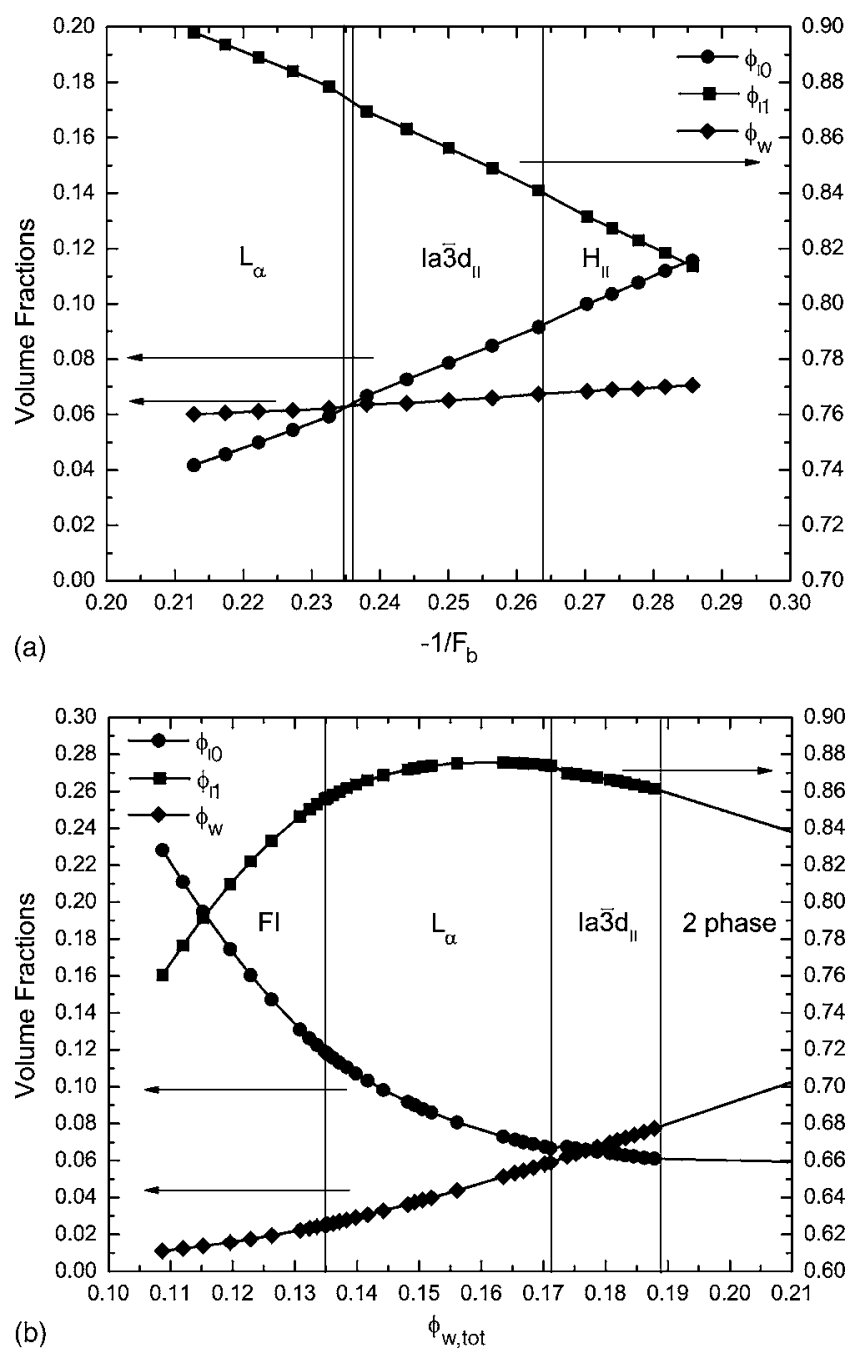

FIG. 4. (a) Volume fractions of free water $\left(\phi_{w}\right)$, unbound lipids $\left(\phi_{l 0}\right)$, and bound lipids $\left(\phi_{l 1}\right)$ vs $-1 / F_{b}$ at the fixed total water content of $\phi_{w, \text { tot }}$ $=0.1750 \pm 0.0003$ and using the model parameters of Fig. 2(b). (b) Volume fractions versus $\phi_{w, \text { tot }}$ at fixed $-F_{b}=4.2$ and using the model parameters of Fig. 2(b).

ter volume fraction) of the gyroid phase stability region $\left(I a \overline{3} d_{\text {III }}\right)$. Thus, while we were unable to find a region of stability for the double diamond phase in our mean-field calculations, the sequence $I a \overline{3} d_{\mathrm{II}} \rightarrow P n \overline{3} m_{\mathrm{II}}$ with increasing water fraction found experimentally is at least partially supported by our model. Examples of the simulated $H_{\mathrm{II}}, I a \overline{3} d_{\mathrm{II}}$, and $P n \overline{3} m_{\mathrm{II}}$ structures are shown in Fig. 5.

\section{B. Lattice parameters and radii of water channels}

Another experimental data set, which can be compared with results from our model, is the lattice parameters for the various structured mesophases. In Fig. 6(a), we show SCFT lattice constants for the inverted hexagonal, gyroid, and double diamond phases, $d_{i}$, expressed in units of a reference lamellar spacing, $d_{L 1}$. The reference point was a lamellar spacing computed for the model system shown in Fig. 2(b) using parameters of $-F_{b}=4.2$ and $\phi_{w, \text { tot }}=0.1353$. These parameters approximately correspond to the experimental conditions under which the lattice constant of the $L_{\alpha}$ phase was determined by x-ray diffraction $\left(45^{\circ} \mathrm{C}\right.$ and $\left.\phi_{w \text {,tot }} \sim 0.135\right) .{ }^{10}$

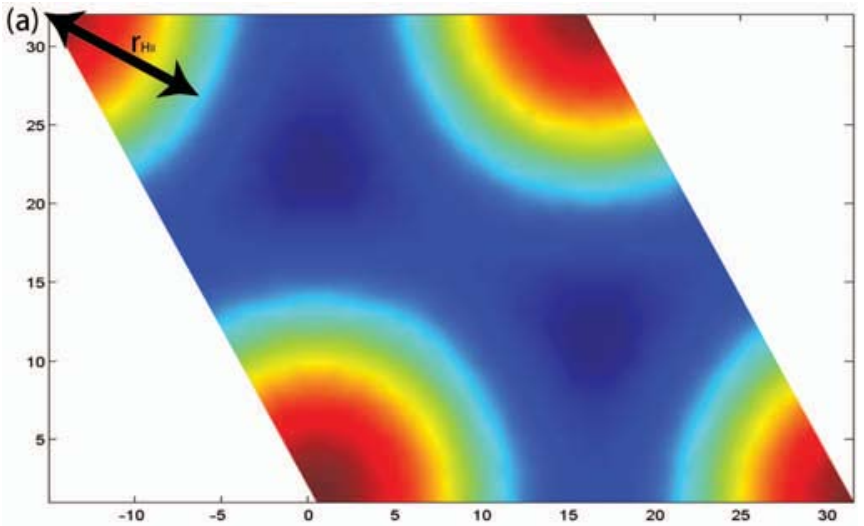

(b)

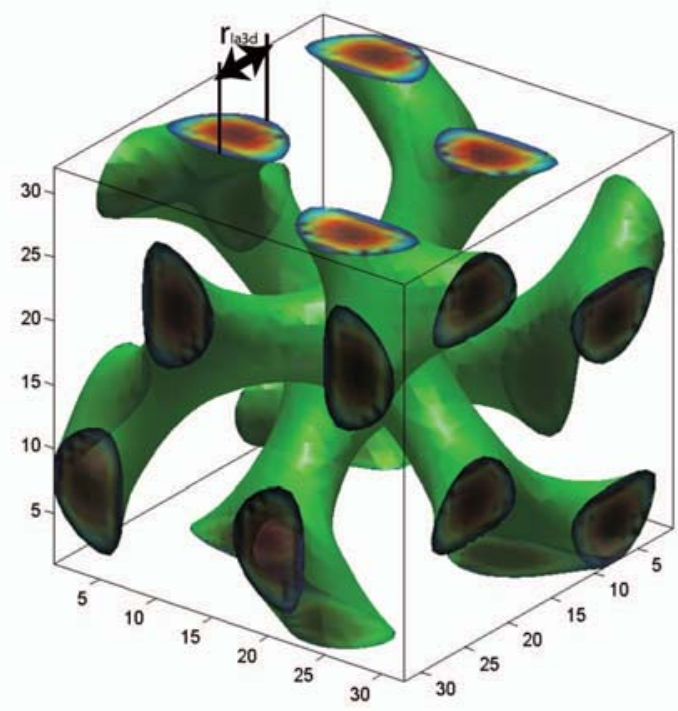

(c)

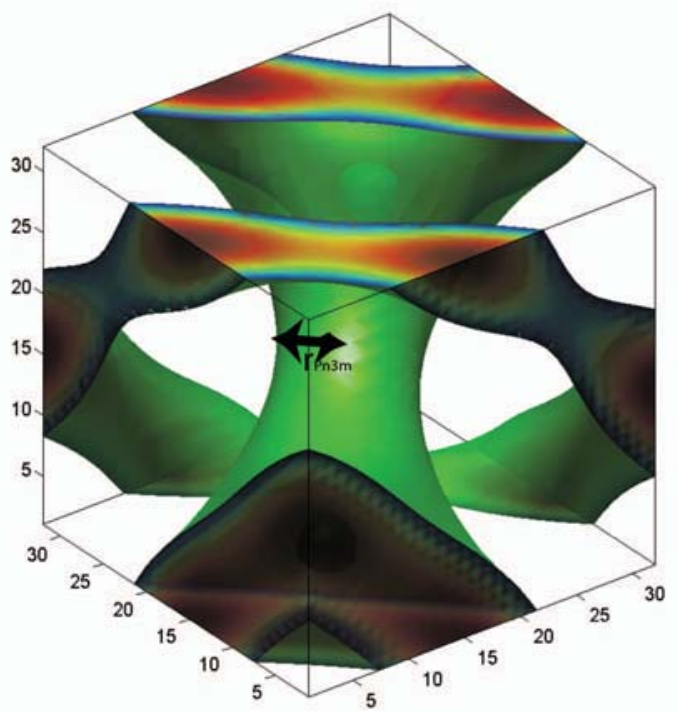

FIG. 5. (Color) (a) Two-dimensional (2D) contour plot for the volume fraction of a $H_{\text {II }}$ phase. (b) Three-dimensional (3D) isosurface plot for the volume fraction of a $I a \overline{3} d_{\text {II }}$ phase. (c) $3 \mathrm{D}$ isosurface plot for the volume fraction of a $P n \overline{3} m_{\text {II }}$ phase. The radii of the water channels for each phases, highlighted by the arrows, are estimated as in Refs. 32 and 42 and shown in Figs. 6 and 7. Excellent agreement is found between topological models and SCFT density profiles. 

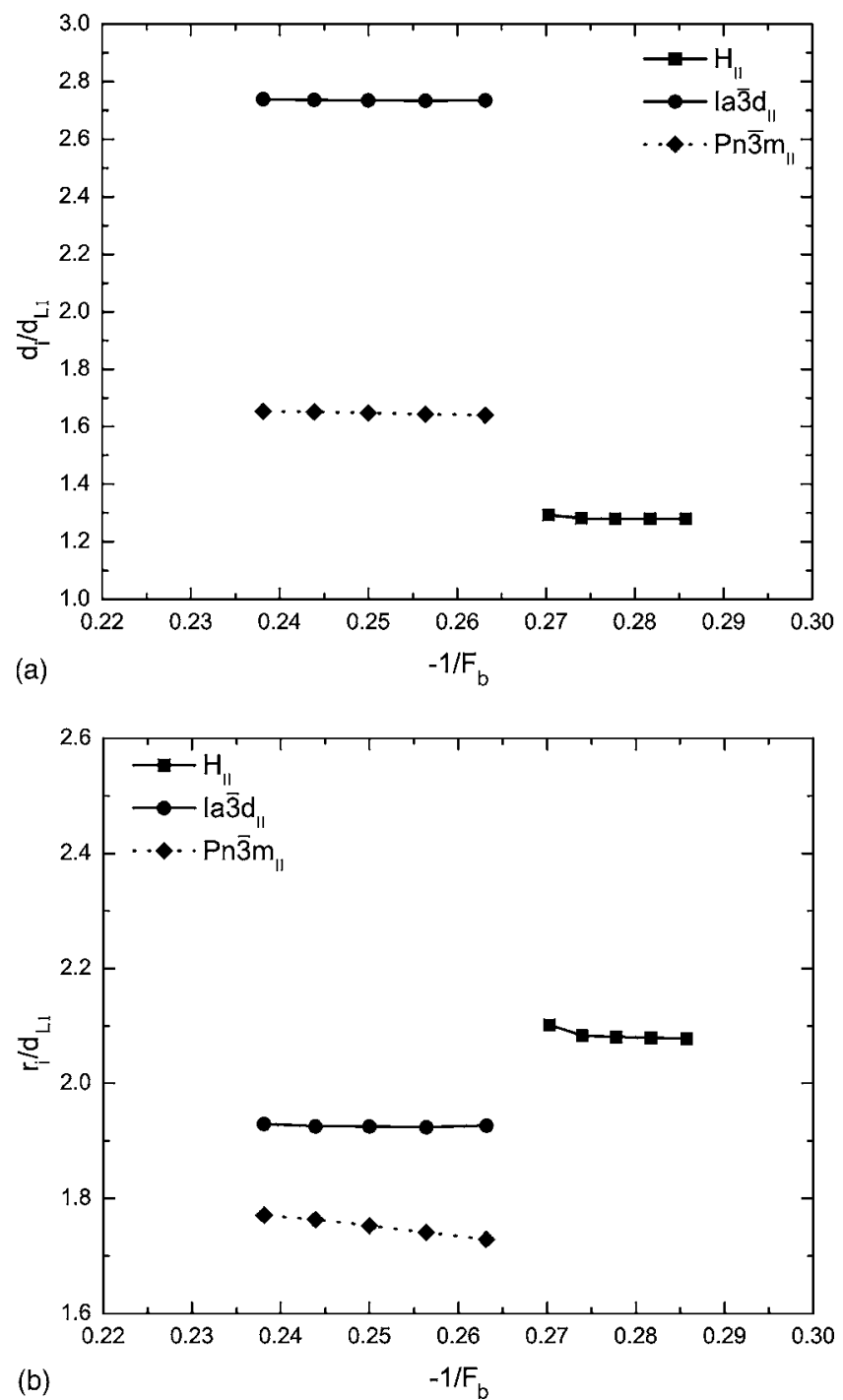

FIG. 6. (a) SCFT predictions for the relative lattice parameters of the hexagonal and cubic mesophases $d_{i} / d_{L 1}$ vs $-1 / F_{b}$ at $\phi_{w, \text { tot }}=0.1750 \pm 0.0003$. The dotted line represents the metastable $P n \overline{3} m_{\text {II }}$ phase. The reference lattice parameter of the lamellar phase $d_{L 1}$ was computed using model parameters $\left(-F_{b}=4.2, \phi_{w, \text { tot }}=0.1353\right)$ in the phase diagram of Fig. 2(b) that best correspond to experimental conditions. (b) SCFT predictions for the relative water channel radii of the hexagonal and cubic mesophases $r_{i} / d_{L 1} \mathrm{vs}-1 / F_{b}$ at $\phi_{w, \text { tot }}=0.1750 \pm 0.0003$. The dotted line represents the metastable $P n \overline{3} m_{\mathrm{II}}$ phase. The reference lamellar spacing $d_{L 1}$ was determined as in (a).

We see from the figure that the predicted lattice parameters have a very weak dependence on temperature $\left(\right.$ or $\left.F_{b}\right)$ but a strong dependence on the symmetry of the mesophase. The SCFT lattice parameters, averaged over the range of $F_{b}$ applied in the simulations, are compared with the experimental values determined by the groups of Caffrey and Mezzenga ${ }^{11,32}$ in Table I. The simulated lattice parameters

TABLE I. Comparison of the lattice parameters of the hexagonal and cubic mesophases normalized by the $L_{\alpha}$ lamellar phase spacing, $d_{i} / d_{L}$, from experiment (Refs. 32 and 42) and from SCFT simulations [cf. Fig. 6(a)].

\begin{tabular}{lccc}
\hline \hline$d_{i} / d_{L}$ & $H_{\mathrm{II}}$ & $I_{a} \overline{3} d_{\mathrm{II}}$ & $P_{n} \overline{3} m_{\mathrm{II}}$ \\
\hline Expt. & 1.36 & 3.11 & 1.89 \\
Simulations & 1.28 & 2.74 & 1.65 \\
\hline \hline
\end{tabular}

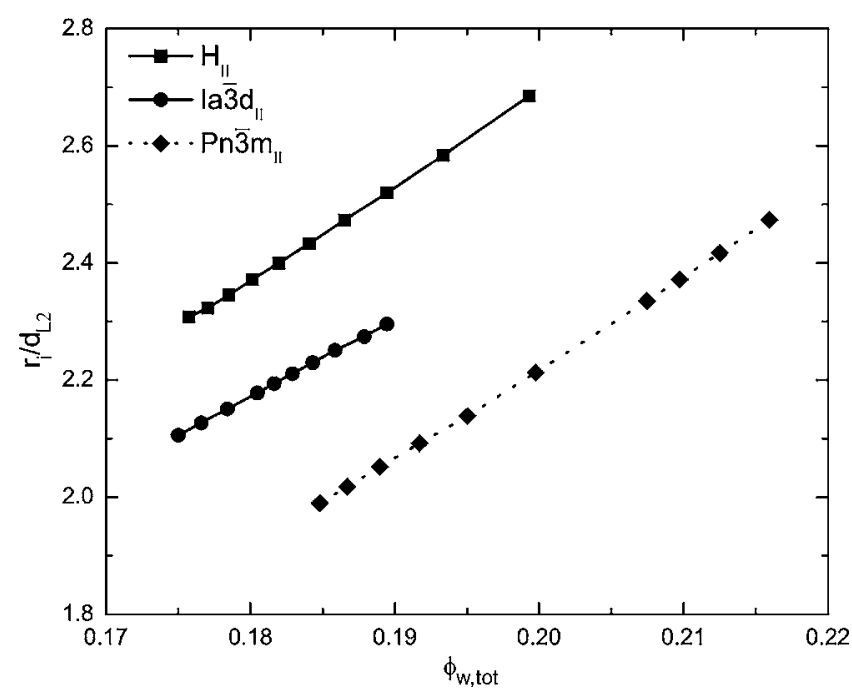

FIG. 7. SCFT predictions for the relative water channel radii $r_{i} / d_{L 2}$ vs $\phi_{w \text {,tot }}$ at $-F_{b}=4.2$ for the cubic mesophases $\left(\operatorname{Ia} \overline{3} d_{\mathrm{II}}\right.$ and $\left.P n \overline{3} m_{\mathrm{II}}\right)$ and at $-F_{b}=3.4$ for the $H_{\text {II }}$ phase. The dotted line represents the metastable $P n \overline{3} m_{\text {II }}$ phase. The model was parametrized as in Fig. 2(b), and the reference lamellar spacing $d_{L 2}$ was obtained from simulations at $-F_{b}=4.7$ and $\phi_{w, \text { tot }}=0.1245$.

fall slightly below the experimental values, although the absolute values of the SCFT predictions for the ratio $d / d_{L}$ and the predicted trends with space group are remarkably good. The under prediction of the lattice constants is apparently related to the total water volume fractions, since the simulated mesophases are somewhat under-hydrated in comparison with the experiment.

Figures 6(b) and 7 show our SCFT model predictions for the radius of the water channels in the hexagonal and cubic mesophases, relative to the lattice parameter of a reference $L_{\alpha}$ phase. The reference lamellar spacing applied in Fig. 6(b), $d_{L 1}$, is the same as that used in Fig. 6(a), while the reference lamellar phase applied in Fig. 7, $d_{L 2}$, was selected as a point in Fig. 2(b) that most closely matched the corresponding experimental conditions $\left(35^{\circ} \mathrm{C}\right.$ and $\left.\phi_{w, \text { tot }} \sim 0.118\right) .{ }^{10}$ The radii of water channels in the simulated phases were calculated using the same procedure as in the experiment. ${ }^{32,42}$ Overall, the SCFT predictions for the water channel radii shown in Figs. 6(b) and 7 are in excellent qualitative agreement with the experiment results. The largest channels are found in the inverted hexagonal phase, while the water channels in the $P n \overline{3} m_{\text {II }}$ phase are the narrowest of the two cubic phases. The channel radii have a very weak temperature dependence, but a strong dependence on both the symmetry of the structure and the total water content.

While the overall trends of water channel radius with topology and water concentration are in agreement with experiment, a discrepancy arises when crossing the $I a \overline{3} d_{\text {II }}$ to $\operatorname{Pn} \overline{3} m_{\text {II }}$ phase boundary by increasing the water volume fraction. Experimentally, no discontinuity in the channel radius is detectable, even though a discontinuity in the lattice constant and channel radius at such a first-order (order-order) phase transition might be expected. However, Fig. 7 suggests such a discontinuity in radius based on the comparison of SCFT 
predictions of the $I a \overline{3} d_{\mathrm{II}}$ and $P n \overline{3} m_{\mathrm{II}}$ phases. We attribute this discrepancy between theory and experiment to the metastability of our simulated $P n \overline{3} m_{\mathrm{II}}$ structure.

\section{Parametric sensitivity of the phase diagrams}

The SCFT calculations reported here are very computationally demanding, so a comprehensive exploration of the (large) parameter space of the model would be an expensive undertaking. Nonetheless, we have conducted a preliminary investigation on the sensitivity of the SCFT phase diagram shown in Fig. 2(b) to the selected parameters. Firstly, if the ratio of bonding to interaction energy $C / B$ is decreased in the model, which increases the rate at which the $\chi_{i, j}$ interaction parameters change with temperature relative to the bonding strength $F_{b}$, the slopes of the phase boundaries between $F I$ and $H_{\mathrm{II}}$ and between $F I$ and $L_{\alpha}$ is diminished. This arises from the increased incompatibility (and concomitant stabilization of ordered mesophases) associated with the increase of $\chi_{i, j}$ upon lowering the temperature. The number of tail segments $N$ is another important parameter. As $N$ is increased at fixed $v_{t} / v_{w}=1.0$, which corresponds to a smaller water molecule relative to the lipid volume of $N v_{t}+v_{h 0}$, the envelope of mesophases widens. This is due to an increase in the translational entropy of the water relative to the lipid, which favors mesophase swelling.

Another important parameter is the ratio of the volume of an unbound lipid head to the volume of a water molecule, $v_{h 0} / v_{w}$. If the value of $v_{h 0} / v_{w}$ is too small, such as $v_{h 0} / v_{w}$ $=1.4$ with $N=6$, the lyotropic $F I$ to $L_{\alpha}$ phase transition disappears. Instead, the system has only a $F I$ to $H_{\text {II }}$ phase transition. Evidently, when the head group is too small, the tail entropy destabilizes the lamellar phase in favor of a phase where the curvature is favorably to the hydrocarbon tails. The nonbonded hydrophobic interaction parameter between the lipid tail and water $\chi_{t, w}$ is also a key parameter. A large value of $\chi_{t, w}$ gives more stability to the $I a \overline{3} d_{\text {II }}$ phase since it increases hydrophobicity, although it also shifts all the mesophases to lower water content. Conversely, if we decrease the value of $\chi_{t, w}$ by $6.4 \%$ at $-F_{b}=4.2$, the $I a \overline{3} d_{\text {II }}$ phase is destabilized relative to $L_{\alpha}$ at all water concentrations. Finally, the value of the hydrated lipid head-water interaction is also important to achieving the topology of the phase diagram shown in Fig. 2(b). When $\chi_{h 1, w}$ is increased, there is diminished contact between free water and the hydrated head groups and correspondingly increased contact between free water and the bare head groups. This promotes head group water binding and drives down the concentration of free water. Therefore, increasing $\chi_{h 1, w}$ decreases the free water content, which decreases the tendency for curvature of bilayers toward water to form cubic and hexagonal phases. Indeed, if we increase the value of $\chi_{h 1, w}$ by $19.5 \%$ at $-F_{b}=4.2$, only the $L_{\alpha}$ phase is stable across all water concentrations.

\section{SUMMARY AND CONCLUSION}

A model of nonionic lipid-water mixtures has been developed in the framework of SCFT, which allows for thermally reversible hydrogen bonding between the lipid head and the surrounding water molecules. Using this model in a two-state approximation for head group hydration, we investigated phase diagrams for two different parametrizations. In the first case, all nonbonded interaction parameters (Flory $\chi$ parameters) were fixed, while the bonding strength in units of the thermal energy $k_{B} T$ was varied. In the second parametrization, we fixed the ratio of bonding to interaction energy $C / B$, while varying the temperature-like variable $-1 / F_{b}$. The second phase diagram more realistically describes temperature effects, and the simulated diagram qualitatively reproduces both the lyotropic and thermotropic phase behaviors observed in the experiments by the groups of Caffrey and Mezzenga. Our conclusions are summarized below.

- The anomalous "reverse" lyotropic phase sequence $\left(L_{\alpha}\right.$ to $I a \overline{3} d_{\mathrm{II}}$ ) observed in the monoolein-water class of systems results from a competition between hydrogen bond formation, changes in head volume and interactions, lipid tail entropy, and the hydrophobic effect. This transition is initiated by the strong hydrophobic interaction between lipid tails and free water. As the water volume fraction is increased, the $L_{\alpha}$ to $I a \overline{3} d_{\text {II }}$ transition serves to reduce the interfacial area between hydrophilic and hydrophobic domains while also increasing the conformational entropy of the lipid tails. The interactions between the hydrated lipid heads and water are also key to obtaining the reverse phase sequence since these serve to mediate the hydrophobic effect.

- The mesophase lattice parameters and the water channel radii calculated by numerical SCFT are in semiquantitative agreement with the experimental values based on $\mathrm{x}$-ray diffraction. Trends with mesophase symmetry and water content are remarkably well reproduced. The SCFT-derived lattice parameters and radii are slightly smaller than their experimental counterparts since the model under predicts the water content of the mesophases.

- The hydrophobic interaction plays a primary role in stabilizing the inverted gyroid phase according to our sensitivity analysis of the model parameters. In addition, the value of the hydrated lipid head volume relative to the tail volume and the molecular volume of water is important in order to obtain the experimentally observed $F I$ to $L_{\alpha}$ transition with increasing water concentration.

- The thermotropic transitions in the monoolein class of lipid-water systems can be explained by thermally induced changes in lipid head volume associated with the binding or unbinding of water, acting in concert with the familiar competition between entropy (translational and conformational) and various nonbonded interactions.

Finally, we point out that SCFT is a field-theoretic model which is solved within the mean-field approximation. The mean field approximation is based on the assumption that one specific configuration of fields dominates the partition function and all other configurations of fields (thermal fluc- 
tuations) are neglected. Therefore, the phase diagrams are supposed to depend on ignored fluctuation effects. For instance, the order-disorder boundaries in the phase diagrams will be shifted to the right since fluctuations stabilize disordered phase. ${ }^{43}$ Fortunately, more advanced field theoretic simulation (FTS) is available, ${ }^{23,26}$ which provides numerical method for the exact field theory although this computationally demanding method has not yet been applied to our model for lipid/water mixtures.

\section{ACKNOWLEDGMENTS}

The authors are grateful to R. Elliott and K. Katsov for useful discussions. This work was supported by the MRSEC Program of the National Science Foundation under Award No. DMR05-20415 and by funding provided to the Complex Fluids Design Consortium at UCSB by the Nestle Research Center.

\section{APPENDIX: VARIABLE CELL SHAPE METHOD}

The variable cell shape method for SCFT is designed in order to obtain stress-free structures in a $n \tau T$ ensemble, ${ }^{39}$ where the thermodynamic tension tensor $\tau$ can be related to an external stress. One variant of the method restricts changes in cell shape to a constant volume manifold in order to preserve the constraint of incompressibility. A second procedure, adopted here in the grand canonical ensemble, involves the minimization of an intensive energy density with respect to the size and shape of a unit cell while maintaining a constant segment density. ${ }^{23}$ Following Refs. 23 and 39, a cell shape tensor $\mathbf{h}$ is introduced, whose components are the three vectors that define the edges of a parallelepiped simulation box. The box shape is relaxed by the following fictitious dynamics scheme:

$$
\frac{d}{d t} \mathbf{h}=-\lambda_{h} \mathbf{h}\left(\sum_{j=0}^{k} \Sigma_{j}[\mathbf{W}, \mathbf{g}]\right),
$$

where $\mathbf{g}$ is a metric tensor defined as $\mathbf{g} \equiv \mathbf{h}^{T} \mathbf{h}$ and $\lambda_{h}$ is a relaxation parameter. The internal stresses produced by lipids in hydration state $j$ are denoted by $\Sigma_{j}$. These internal stresses vanish at equilibrium and can be evaluated in terms of propagators in the grand canonical ensemble by invoking a factorization of the single chain path integrals ${ }^{23,39}$

$$
\begin{aligned}
\Sigma_{j}[\mathbf{W}, \mathbf{g}]= & 2 g_{j, k} z_{h j} \\
& \times \int d \mathbf{X} \int_{0}^{1} d s q_{h j}(\mathbf{X}, s) \mathbf{g}^{-1} \nabla_{\mathbf{X}} \nabla_{\mathbf{X}} \mathbf{g}^{-1} q_{t}(\mathbf{X}, 1-s) .
\end{aligned}
$$

In these expressions, $\mathbf{X}=\mathbf{h}^{-1} \cdot \mathbf{r}$ is a cell-scaled position vector whose components lie in $[0,1]$.
${ }^{1}$ S. A. Walker, M. T. Kennedy, and J. A. Zasadzinski, Nature (London) 387, 61 (1997).

${ }^{2}$ I. Koltover, T. Salditt, J. O. Rädler, and C. R. Safinya, Science 281, 78 (1998).

${ }^{3}$ D. E. Discher and A. Eisenberg, Science 297, 967 (2005).

${ }^{4}$ R. Mezzenga, P. Schurtenberger, A. Burbidge, and M. Michel, Nat. Mater. 4, 729 (2005).

${ }^{5}$ V. Luzzati and P. A. Spegt, Nature (London) 215, 701 (1967).

${ }^{6}$ L. E. Scriven, Nature (London) 263, 123 (1976).

${ }^{7}$ W. Longley and T. J. McIntosh, Nature (London) 203, 612 (1983).

${ }^{8}$ K. Larsson, Nature (London) 304, 664 (1983).

${ }^{9}$ S. T. Hyde, S. Andersson, B. Ericsson, and K. Larsson, Z. Kristallogr. 168, 213 (1984).

${ }^{10}$ J. Briggs, H. Chung, and M. Caffrey, J. Phys. I 6, 723 (1996).

${ }^{11} \mathrm{H}$. Qiu and M. Caffrey, Biomaterials 21, 223 (2000).

${ }^{12}$ H. Qiu and M. Caffrey, J. Phys. Chem. B 102, 4819 (1998).

${ }^{13}$ Y. Misquitta and M. Caffrey, Biophys. J. 81, 1047 (2001).

${ }^{14}$ J. Israelachvili, Intermolecular and Surface Forces, 2nd ed. (Academic, New York, 1991).

${ }^{15}$ P. B. Canham, J. Theor. Biol. 26, 61 (1970).

${ }^{16}$ W. Helfrich, Z. Naturforsch. C 28, 693 (1973).

${ }^{17}$ U. S. Schwarz and G. Gompper, Phys. Rev. Lett. 85, 1472 (2000).

${ }^{18}$ M. A. Wilson and A. Pohorille, J. Am. Chem. Soc. 116, 1490 (1994).

${ }^{19}$ S. J. Marrink and D. P. Tieleman, J. Am. Chem. Soc. 123, 12383 (2001).

${ }^{20}$ E. Lindahl and O. Edholm, Biophys. J. 79, 426 (2000).

${ }^{21}$ S. J. Marrink and A. E. Mark, J. Phys. Chem. B 105, 6122 (2001).

${ }^{22}$ M. W. Matsen and M. Schick, Curr. Opin. Colloid Interface Sci. 1, 329 (1996).

${ }^{23}$ G. H. Fredrickson, The Equilibrium Theory of Inhomogeneous Polymers (Oxford University Press, Oxford, 2006).

${ }^{24}$ M. W. Matsen and M. Schick, Phys. Rev. Lett. 72, 2660 (1994).

${ }^{25}$ M. W. Matsen, Phys. Rev. Lett. 74, 4225 (1995).

${ }^{26}$ G. H. Fredrickson, V. Ganesan, and F. Drolet, Macromolecules 35, 16 (2002).

${ }^{27}$ R. Mezzenga, W. B. Lee, and G. H. Fredrickson, Trends Food Sci. Technol. 17, 220 (2006).

${ }^{28}$ X. Li and M. Schick, Biophys. J. 78, 34 (2000).

${ }^{29}$ P. J. Flory, Statistical Mechanics of Chain Molecules (Wiley-Interscience, New York, 1969).

${ }^{30}$ M. Müller and M. Schick, Phys. Rev. E 57, 6973 (1998).

${ }^{31}$ W. B. Lee, R. Mezzenga, and G. H. Fredrickson, Phys. Rev. Lett. 99, 187801 (2007).

${ }^{32}$ R. Mezzenga, C. Meyer, C. Servais, A. I. Romoscanu, L. Sagalowicz, and R. C. Hayward, Langmuir 21, 3322 (2005).

${ }^{33}$ E. H. Feng, W. B. Lee, and G. H. Fredrickson, Macromolecules 40, 693 (2007).

${ }^{34}$ W. B. Lee, R. Elliot, K. Katsov, and G. H. Fredrickson, Macromolecules 40, 8445 (2007).

${ }^{35}$ G. Tzeremes, K. O. Rasmussen, T. Lookman, and A. Saxena, Phys. Rev. E 65, 041806 (2002).

${ }^{36}$ K. O. Rasmussen and G. Kalosakas, J. Polym. Sci., Part B: Polym. Phys. 40, 1777 (2002).

${ }^{37}$ H. D. Ceniceros and G. H. Fredrickson, Multiscale Model. Simul. 2, 452 (2004).

${ }^{38}$ S. W. Sides and G. H. Fredrickson, Polymer 44, 5859 (2003).

${ }^{39}$ J. L. Barrat, G. H. Fredrickson, and S. W. Sides, J. Phys. Chem. B 109, 6694 (2005).

${ }^{40}$ H. J. Angerman and G. ten Brinke, Macromolecules 32, 6813 (1999).

${ }^{41}$ V. Kocherbitov, J. Phys. Chem. B 109, 6430 (2005).

${ }^{42}$ R. Mezzenga, M. Grigorov, Z. Zhang, C. Servais, L. Sagalowicz, A. I. Romoscanu, V. Khanna, and C. Meyer, Langmuir 21, 6165 (2005).

${ }^{43}$ G. H. Fredrickson and E. Helfand, J. Chem. Phys. 87, 697 (1987). 